\title{
Homonymous hemianopia: An unusual presentation of vitamin B 12 deficiency
}

\section{Ravindra Kumar Chowdhury}

Assistant Professor, VSS Institute of Medical Science and Research, Burla, Odisha, India

*Corresponding Author: Ravindra Kumar Chowdhury

Email: ravindrachowdhury@gmail.com

\begin{abstract}
A 24 years male presented with a headache for one year, recent memory loss and loss of left hemifield of vision for three months. Upon examination, visual acuity was found to be $20 / 20$ in both eyes. Automated perimetry revealed left homonymous hemianopia with sparing of the macula. Right thalamic infarct and subacute infarct in the territory of the right posterior cerebral artery (PCA) were the findings on CT scan. Except for decreased vitamin B12 level and increased homocysteine level in serum, rest of the serological, biochemical, hormonal and immunological investigations were within normal limits. MRI of the brain showed right PCA infarct and few ischemic foci in bilateral periventricular regions. MR Angiogram revealed relatively reduced flow in the right PCA and right vertebral artery. The patient was managed with antiplatelet drugs and supplementation of vitamin B12. Marked improvement was noticed in a headache and memory loss and there was normalization of field defect after 6 months of treatment. Cases of homonymous hemianopia in neuro-ophthalmology are not unusual, but the etiology and course of this case report make an interesting variation.
\end{abstract}

Keywords: Homonymous hemianopia, Vitamin B12 deficiency, Posterior Cerebral artery infarct.

\section{Introduction}

Homonymous hemianopia in adults most commonly results due to vascular causes like posterior cerebral artery (PCA) occlusion and intracranial hemorrhage at occipital lobe followed by brain tumors, trauma, surgical interventions and other central nervous system diseases., ${ }^{1,2}$ The common etiologies of PCA occlusion are cardiac emboli, atheromatous plaque, and dissection of artery proximal to PCA. ${ }^{3}$ Migrainous infarction, hypercoagulable disorders, illicit substance use, vasculitis, and fibromuscular dysplasia are less common causes. ${ }^{3}$ However infarct at PCA territory due to vitamin B12 deficiency which improves on supplementation is rare as per available literature.

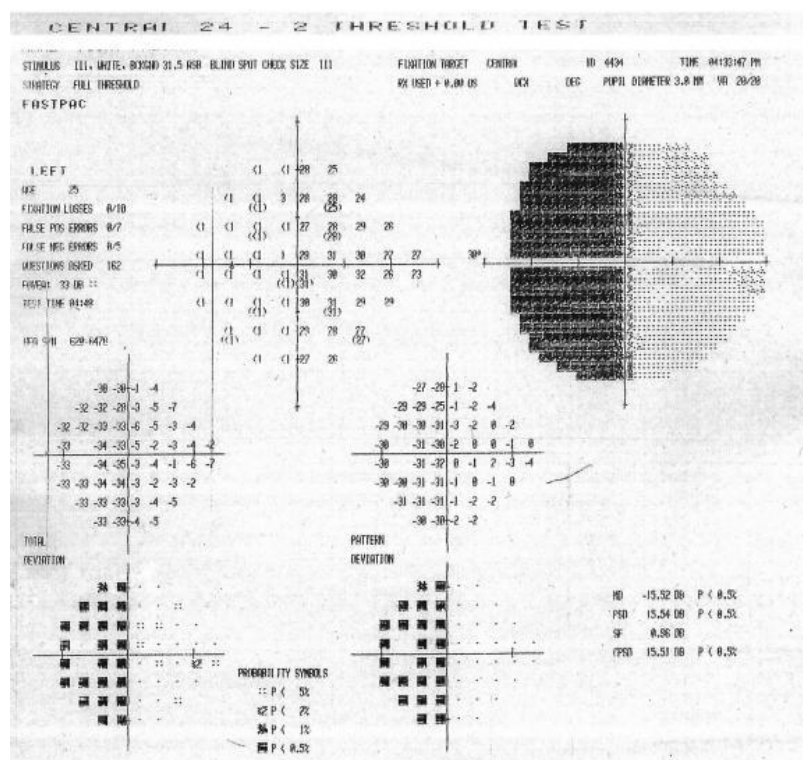

\section{Case Report}

A 24 years male presented with a headache for one year, recent memory loss and loss of left hemifield of vision for three months. The patient did not complain of fever or vomiting. There was no history of seizures or loss of consciousness. History of trauma was absent.

On local examination, orbital margins were intact. Extraocular movements were full in all directions of gaze. The lid and conjunctiva were normal. Both the direct and consensual light reflex was present. Other anterior segments and posterior segment details were within normal limits. The visual acuity was 20/20 in both eyes.

The automated perimetry examination showed left homonymous hemianopia with sparing of the macula. [Fig. $1]$.

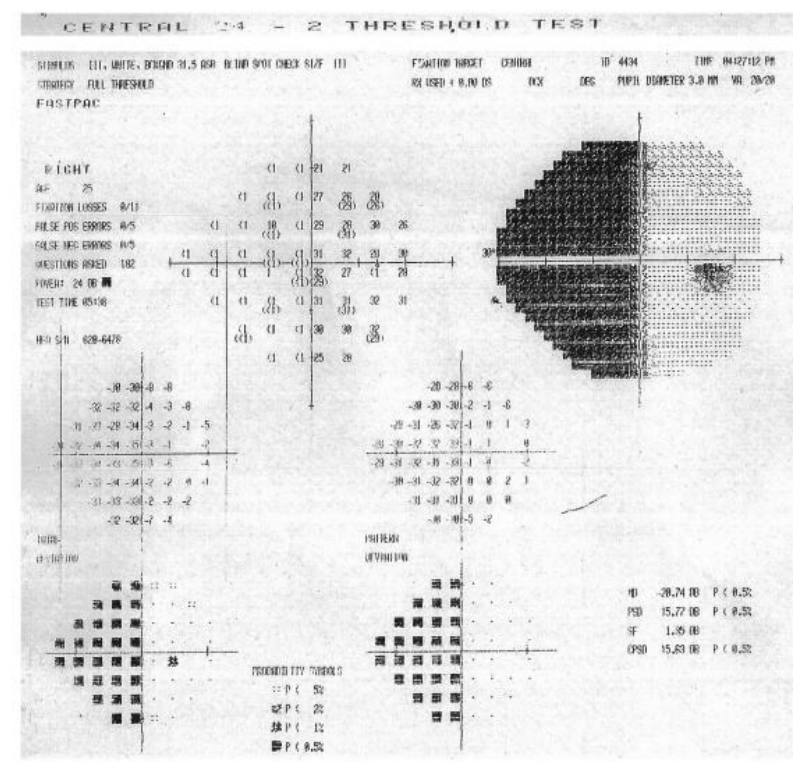

Fig. 1: Automated perimetry showing left homonymous hemianopia 
The serological, biochemical, immunological and hormonal investigations like complete haemogram, fasting blood sugar, liver function test, renal function test, thyroid function test, lipid profile, sickling test, protein c, protein $S$, Antithrombin III, lupus anticoagulant, LE cell, prothrombin time, activated partial thromboplastin time, apolipoprotein A1, apolipoprotein B, lipoprotein A, total iron, total ironbinding capacity, percentage transferrin saturation, RA factor, ANA test were done. All the parameters were found to be within normal range except features of megaloblastic anemia in complete haemogram. Serum Vitamin B12 level was found to be less than $30 \mathrm{pg} / \mathrm{ml}$ (Normal-211-946 pg/ml) and serum homocysteine level was found to be raised (Normal-4-15 micromoles/liter).

CT scan of the brain revealed right posterior cerebral artery subacute infarct and right thalamic lacunar infarct. [Fig. 2]. MRI of the brain showed chronic right posterior cerebral artery infarct and few ischemic foci in the bilateral periventricular region [Fig. 3]. MR angiogram of the intracranial region detected relatively reduced flow in the right posterior cerebral artery and right vertebral artery. ECG and echocardiogram were normal.

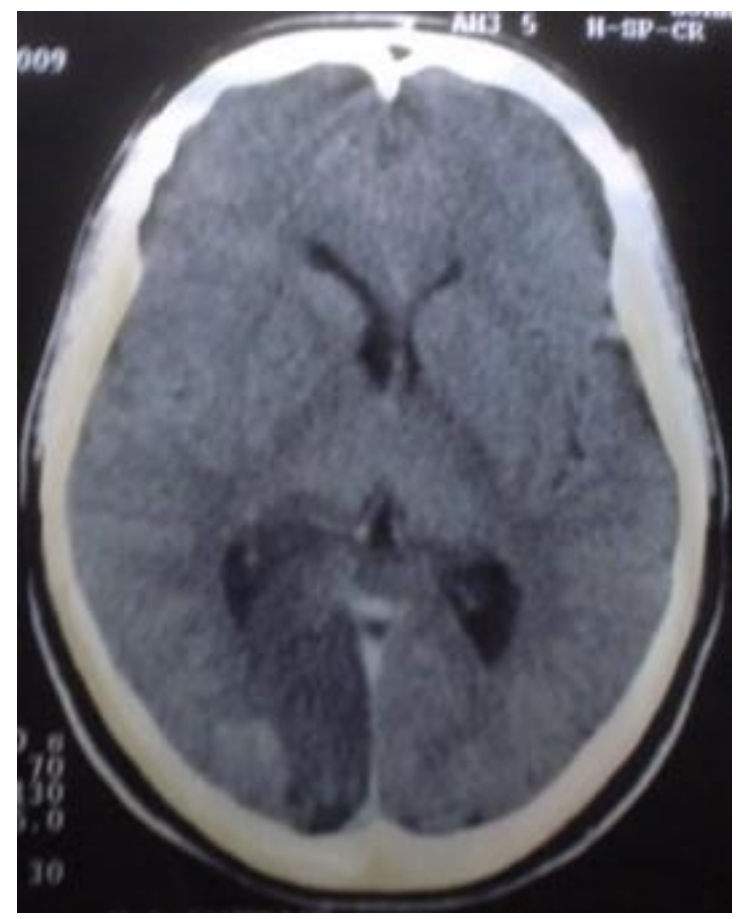

Fig. 2: CT scan of the brain showing infarct at right PCA territory

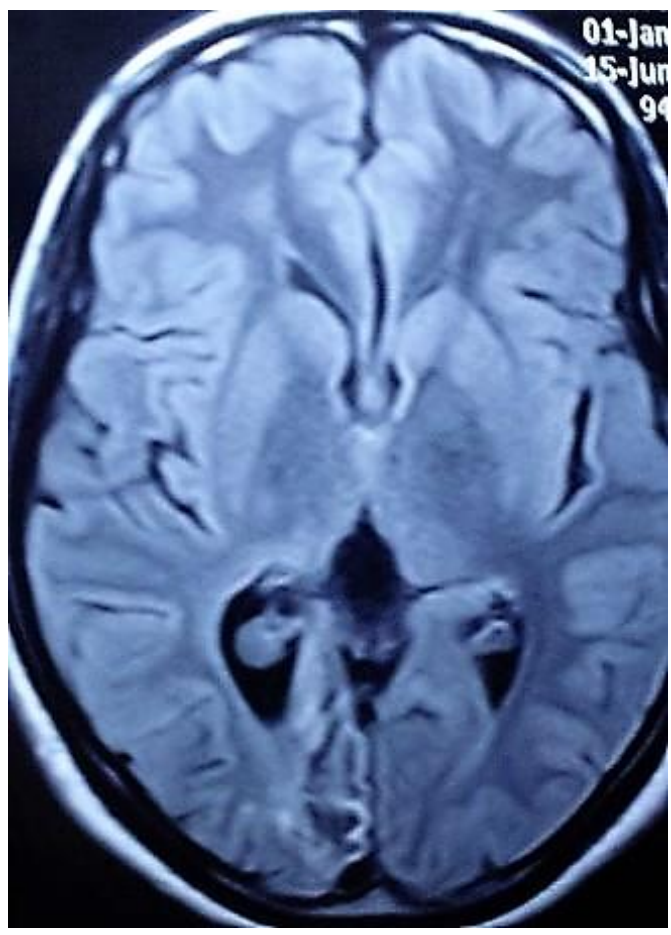

Fig. 3: MRI of the brain showing hyperintense lesion at the right occipital lobe

As the etiology behind the left homonymous hemianopia could not be delineated except decreased vitamin B12 and increased homocysteine level in serum, the case was managed with supplementation of vitamin B12 and antiplatelet drugs. Marked improvement of a headache, recent memory and normalization of field defect was seen after 6 months follow up.

\section{Discussion}

This case presents the clinical course and evaluation of a young patient presenting with homonymous hemianopia. The homonymous hemianopia due to posterior cerebral artery territory (PCA) infarct is not so unusual, but the infarct due to vitamin B12 deficiency which improves on treatment with vitamin B12 is a very rare interesting variation. The disconnection syndrome of PCA territory infarct of dominant hemisphere due to vitamin B12 deficiency has been reported in the literature. ${ }^{4}$ Verma et al in their case report found Alexia as an additional symptom due to infarction of spenicial part of the corpus callosum of dominant side. ${ }^{4}$ But in our case as the involvement of infarct is on the nondominant side, we did not encounter any such symptoms. However recent memory loss in our case is probably attributed to the presence of thalamic infarct. Infarction of medial temporal lobe, fornices or medial thalamic nuclei may result in permanent antegrade amnesia. ${ }^{5}$ Malnutrition including, vitamin deficiencies like folate and vitamin $\mathrm{B} 12$ has been postulated as a predisposing cause of splenial lesions of the corpus callosum. ${ }^{6}$ Kori described a patient with B12 deficiency having reversible splenial lesion not associated with any disconnection syndrome. ${ }^{7}$ Verma et al have reported 
complete improvement in symptoms following administration vitamin B12. In our case also homonymous hemianopia improved completely. Early administration of vitamin B12 plays a vital role in the improvement of symptoms. Our case report was consistent with that of Penix's report regarding the finding of megaloblastic anemia, hyperhomocysteinemia and decrease vitamin B12 level. ${ }^{8}$ However the cause of vitamin B12 deficiency was ileal resection in their report, but in our case, it was probably due to dietary deficiency. Band atrophy or bow-tie atrophy of optic disc is seen in homonymous hemianopia due to the lesion at optic tract. ${ }^{9}$ This band atrophy is seen contralateral to the side of the lesion. However, in our case, we did not found any such finding as the lesion was posterior to the lateral geniculate body.

Ischemic stroke occurs when cerebral blood flow to a region is suddenly impaired. This may occur either by vessel occlusion or by the relatively low blood flow. The neuronal death rates of an individual varies with blood flow, collateralization of blood vessel and inherent cerebral capacities to withstand lack of blood supply. Infarction of the brain tissue is produced, when the cerebral blood flow rate decreases to less than $20 \mathrm{ml} / 100 \mathrm{gm} / \mathrm{min}$. However rapid restoration of blood flow is the most effective means of preservation of brain tissue. In our case probably restoration of blood flow was possible because of early supplementation of vitamin B12.

Methylcobalamin deficiency leads to an increase in serum homocysteine and methylmalonic acid, which is vasculotoxic and neurotoxic resulting in various neurologic complications. Decrease bioavailability of nitric oxide, which is a potent vasodilator is one of the major key events in the production of atherothrombosis in hyperhomocysteinemia. White-matter hyperintensities and areas of demyelination on brain MRI have been described in patients with vitamin B12 deficiency. ${ }^{10}$ Verma et al reported the complete disappearance of the hyperintense lesion in occipital lobe which is consistent with our case.

The detail investigation and early treatment play a major role in the management of homonymous hemianopia.

\section{Conflict of Interest: None.}

\section{References}

1. Levin LA. Topical diagnosis of chiasmal and retrochiasmal disorders. In: Walsh and Hoyt Clinical Neuro-ophthalmology, 6th, Miller NR, Newman NJ, Biousse V, Kerrison JB. (Eds), Williams \& Wilkins, Baltimore 2005. p.503.
2. Zhang X, Kedar S, Lynn MJ, Newman NJ, Biousse V. Homonymous hemianopias: clinical-anatomic correlations in 904 cases. Neurol 2006;66(6):906-10.

3. Yamamoto Y, Georgiadis AL, Chang HM, Caplan LR. Posterior cerebral artery territory infarcts in the New England Medical Center Posterior Circulation Registry. Arch Neurol 1999;56(7):824-32.

4. Verma R, Raut TP, Praharaj HN. Reversible disconnection syndrome: an unusual presentation of vitamin B12 deficiency. Neurol India 2013;61(2):167-9.

5. Peters J, Koch B, Schwarz M, Daum I. Domainspecific impairment of source memory following a right posterior medial temporal lobe lesion. Hippocampus 2007;17(7):505-9.

6. Kosugi T, Isoda H, Imai M, Sakahara H. Reversible focal splenial lesion of the corpus callosum on MR images in a patient with malnutrition. Magn Reson Med Sci 2004;3:211-4.

7. Kori S. Hyperintense splenium in vitamin B12 deficiency. Neurol India 2005;53(3):377-8.

8. Penix LP. Ischemic strokes secondary to vitamin B12 deficiency-induced hyperhomocystinemia. Neurol 1998;51(2):622-4.

9. Gálvez-Ruiz A, Arishi N. Band atrophy of the optic nerve: A report on different anatomical locations in three patients. Saudi J Ophthalmol 2013;27(1):65-9.

10. Vry MS, Haerter K, Kastrup O, Gizewski E, Frings M, Maschke M. Vitamine-B12-deficiency causing isolated and partially reversible leukoencephalopathy. $J$ Neurol 2005;252(8):980-2.

How to cite this article: Chowdhury RK. Homonymous hemianopia: An unusual presentation of vitamin B 12 deficiency. Int J Ocul Oncol Oculoplasty 2019;5(2):97-9. 